\title{
ON WEAKLY $S$-PRIMARY IDEALS OF COMMUTATIVE RINGS
}

\author{
ECE YETKIN CELIKEL AND HANI A. KHASHAN
}

\begin{abstract}
Let $R$ be a commutative ring with identity and $S$ be a multiplicatively closed subset of $R$. The purpose of this paper is to introduce the concept of weakly $S$-primary ideals as a new generalization of weakly primary ideals. An ideal $I$ of $R$ disjoint with $S$ is called a weakly $S$-primary ideal if there exists $s \in S$ such that whenever $0 \neq a b \in I$ for $a, b \in R$, then $s a \in \sqrt{I}$ or $s b \in I$. The relationships among $S$-prime, $S$-primary, weakly $S$-primary and $S$ - $n$-ideals are investigated. For an element $r$ in any general ZPI-ring, the (weakly) $S_{r^{-}}$ primary ideals are charctarized where $S=\left\{1, r, r^{2}, \cdots\right\}$. Several properties, characterizations and examples concerning weakly $S$-primary ideals are presented. The stability of this new concept with respect to various ring-theoretic constructions such as the trivial ring extension and the amalgamation of rings along an ideal are studied. Furthermore, weakly $S$-decomposable ideals and $S$-weakly Laskerian rings which are generalizations of $S$-decomposable ideals and $S$-Laskerian rings are introduced.
\end{abstract}

\section{INTRODUCTION}

This paper is concerned with a new class of ideals of commutative rings. All the rings considered in this paper will be commutative and have non-zero identities. For the sake of completeness, we begin with some definitions and notations that will be followed in this paper. For a ring $R$, we will denote by $U(R), \operatorname{reg}(R)$ and $Z(R)$, the set of unit elements, regular elements and zero-divisor elements of $R$, respectively. For an ideal $I$ of $R$, the radical of $I$ denoted by $\sqrt{I}$ is the ideal $\left\{a \in R: a^{n} \in I\right.$ for some positive integer $n\}$ of $R$. In particular, $\sqrt{0}$ denotes the set of all nilpotent elements of $R$. A non-empty subset $S$ of a ring $R$ is said to be a multiplicatively closed subset of $R$ if $0 \notin S, 1 \in S$ and $s t \in S$ for all $s, t \in S$ [20]. As usual, we denote by $\mathbb{Z}$ and $\mathbb{Z}_{n}$ the ring of integers and the ring of integers modulo $n$ where $n \geq 2$.

In recent years, the prime ideals and their generalizations have an important place in commutative algebra and they draw attention by several authors. In 2003, the concept of weakly prime ideals, which is a main generalization of prime ideals, was first initiated by Anderson and Smith in [3]. They call a proper ideal $I$ of $R$ a weakly prime ideal if whenever $0 \neq a b \in I$ for some $a, b \in R$, then $a \in I$ or $b \in I$. Afterwards, in 2005, Atani and Farzalipour generalized this concept by defining weakly primary ideals. In their celebrated paper [4], a proper ideal $I$ of $R$ is said to be weakly primary if $0 \neq a b \in I$ for some $a, b \in R$ implies $a \in I$ or $b \in \sqrt{I}$. In 2020, Hamed and Malek [12] used a new approach to generalize prime ideals by defining $S$-prime ideals. Then, in a very recent study [1], the notion of weakly $S$-prime

2000 Mathematics Subject Classification. Primary 13A15.

$K e y$ words and phrases. $S$-prime ideal, $S$-primary ideal, weakly $S$-prime ideal, $S$ - $n$-ideal, weakly $S$ - $n$-ideal, weakly $S$-primary ideal.

This paper is in final form and no version of it will be submitted for publication elsewhere. 
ideals is studied. Let $S$ be a multiplicatively closed subset of a ring $R$. An ideal $I$ of $R$ disjoint with $S$ is said to be $S$-prime (resp. weakly $S$-prime) if there exists an $s \in S$ such that for all $a, b \in R$ if $a b \in I$ (resp. $0 \neq a b \in I$ ), then either $s a \in I$ or $s b \in I$. Analogously, Visweswaran [19] introduced the notion of $S$-primary ideals. An ideal $I$ disjoint with $S$ is called $S$-primary ideal of $R$ if there exists an $s \in S$ such that for all $a, b \in R$ if $a b \in I$, then $s a \in I$ or $s b \in \sqrt{I}$. recently, the notion of $S$ - $n$-ideals is introduced by the authors, [13] generalizing $n$-ideals introduced in [18]. An ideal $I$ disjoint with $S$ is called a $S$ - $n$-ideal if there exists $s \in S$ such that for all $a, b \in R$ if $0 \neq a b \in I$ and $s a \notin \sqrt{0}$, then $s b \in I$.

Our purpose here is to introduce weakly $S$-primary ideals generalizing the concept of weakly primary ideals via a multiplicative subset $S$ of a ring. Among other results in this paper, in Section 2, we give many properties and characterizations of this new class of ideals. We also generalize some results on weakly primary ideals to weakly $S$-primary ideals. We describe in detail the behavior of weakly $S$-primary ideals under homomorphic images, localizations, and direct product of rings (see Theorems 2, Propositions 9, 10, 12, Corollaries 2, 4 and Theorem 4, 5). For an element $r$ in a general ZPI-ring $R$, we characterize all (weakly) $S_{r}$-primary ideals of $R$ where $S_{r}=\left\{1, r, r^{2}, r^{3}, \ldots\right\}$, (see Theorem 1). Moreover, we construct several examples to support given propositions. We characterize rings in which the zero ideal is the only weakly $S$-primary ideal (see Theorem 3 ).

In Section 3, we study the stability of this new concept with respect to various ring-theoretic constructions such as the trivial ring extension and the amalgamation of rings along an ideal.

Finally, Section 4 is devoted to introduce weakly $S$-primary decomposable ideals and $S$-weakly Laskerian rings generalizing the concepts of $S$-decomposable and $S$ Laskerian rings introduced in [19]. We explore some relationships between these concepts and provide some examples.

\section{Characterizations of Weakly $S$-Primary ideals}

Definition 1. Let $S$ be a multiplicatively closed subset of a ring $R$ and let $I$ be an ideal of $R$ disjoint with $S$.

(1) $I$ is called a weakly $S$-primary ideal if there exists an (fixed) $s \in S$ such that for all $a, b \in R$ if $0 \neq a b \in I$, then $s a \in \sqrt{I}$ or $s b \in I$. This fixed element $s \in S$ is called a weakly $S$-primary element of $I$.

(2) $I$ is called a weakly $S$-n-ideal if there exists an (fixed) $s \in S$ such that for all $a, b \in R$ if $0 \neq a b \in I$ and $s a \notin \sqrt{0}$, then $s b \in I$. This fixed element $s \in S$ is called a weakly $S$-n-element of $I$.

Clearly, every weakly primary ideal of a ring $R$ is a weakly $S$-primary ideal, and these two concepts coincide if $S \subseteq U(R)$. Also, obviously, every $S$-primary ideal of $R$ is a weakly $S$-primary ideal. The converse is clearly true if $R$ is Domain-like (the zero ideal of $R$ is primary). Moreover, every weakly $S$-prime ideal is a weakly $S$-primary ideal and the converse holds for radical ideals. It is clear that any weakly $S$ - $n$-ideal is a weakly $S$-primary ideal and the two concepts coincide if the ideal is contained in $\sqrt{0}$. In the following diagram, we show the place of weakly $S$-primary ideals among the other ideal structures in the literature of a commutative ring. 


$\begin{array}{ccccc}\text { weakly prime } & \rightarrow & \text { weakly } S \text {-prime } & & S \text {-primary } \\ & \searrow & & & \downarrow \\ & \nearrow & \text { weakly primary } & \rightarrow & \text { weakly } S \text {-primary } \\ \text { weakly } n \text {-ideal } & \rightarrow & \text { weakly } S \text { - } n \text {-ideal } & \longleftarrow & S \text { - } n \text {-ideal }\end{array}$

We give the following examples to show that the converses of some of these implications are not true in general. Counter examples for the rest of implications can be found in [1], [3], [4], [12] and [13].

\section{Example 1.}

(1) Let $p$ be a prime integer. Consider the polynomial ring $R=\mathbb{Z}[X]$ and the multiplicatively closed subset $S=\left\{p^{n}: n \in \mathbb{N}\right\}$ of $R$. Then the ideal $I=p^{3} X \mathbb{Z}[X]$ is a (weakly) $S$-primary ideal of $R$. Indeed, suppose that $0 \neq f \cdot g \in I$ for some $f, g \in R$. Since $I \subseteq X \mathbb{Z}[X]$ and $X \mathbb{Z}[X]$ is prime, we conclude either $f \in X \mathbb{Z}[X]$ or $g \in X \mathbb{Z}[X]$. Put $s=p \in S$. Then $s f \in \sqrt{I}$ or $s g \in \sqrt{I}$, as needed. However, it is not (weakly) primary ideal of $R$ as $0 \neq p \cdot p^{2} \cdot X \in I$ but neither $p \in \sqrt{I}$ nor $p^{2} X \in I$.

(2) Any weakly primary ideal of a ring that is not primary is clearly a weakly $\{1\}$-primary ideal that is not $\{1\}$-primary. For a less trivial example, consider the multiplicatively closed subset $S=\{\overline{1}, \overline{5}, \overline{25}\}$ of the ring $\mathbb{Z}_{30}$. Then the ideal $\langle\overline{0}\rangle$ is a weakly $S$-primary that is not $S$-primary. Indeed $\overline{15} \cdot \overline{2} \in\langle\overline{0}\rangle$ but clearly $s \cdot \overline{15} \notin \sqrt{\langle\overline{0}\rangle}=\langle\overline{0}\rangle$ and $s \cdot \overline{2} \notin\langle\overline{0}\rangle$ for every $s \in S$.

(3) The ideal $\langle\overline{4}\rangle$ is a (weakly) primary ideal of the ring $\mathbb{Z}_{12}$ and so weakly $S$-primary for $S=\{\overline{1}, \overline{9}\}$. On the other hand, $0 \neq \overline{2} \cdot \overline{2} \in\langle\overline{4}\rangle$ but $s \cdot \overline{2} \notin\langle\overline{4}\rangle$ for every $s \in S$. Thus, $\langle\overline{4}\rangle$ is not weakly $S$-prime.

(4) The ideal $\langle\overline{6}\rangle$ is (weakly) $S$-primary in $\mathbb{Z}_{30}$ for $S=\{\overline{1}, \overline{2}, \overline{4}, \overline{8}, \overline{16}\}$, see (2) of Proposition 1. But $\langle\overline{6}\rangle$ is not a (weakly) $S$ - $n$-ideal since for example, $3 \cdot 2 \in\langle\overline{6}\rangle$ but $3 \cdot s \notin\langle\overline{0}\rangle=\sqrt{0}$ and $2 \cdot s \notin\langle\overline{6}\rangle$ for all $s \in S$. Also, while clearly, $\langle\overline{0}\rangle$ is a weakly $S$ - $n$-ideal of $\mathbb{Z}_{30}$, it is not a $S$ - $n$-ideal, [13, Theorem $3]$.

Recall that a ring $R$ is said to be a general ZPI-ring (resp. ZPI-ring) if each of its ideals (resp. non-zero ideals) can be uniquely expressible as product of prime ideals of $R$. Dedekind domains are indecomposable general ZPI-rings. It is known from [10, page 475, Exercise 9] that a Noetherian ring $R$ is a general ZPI-ring if and only if each primary ideal of $R$ is a prime power. For a general background, the reader may refer to [10].

Theorem 1. Let $R$ be a general ZPI-ring, $r \in R$ and $I$ be a non-zero proper ideal of $R$ disjoint with $S_{r}=\left\{r^{n}: n \in \mathbb{N}\right\}$. Then we have the following cases:

(1) If $I=P^{l}$ for some prime ideal $P$ of $R$ and $r \notin P$, then $I$ is a (weakly) $S_{r}$-primary ideal of $R$.

(2) If $I=P_{1}^{t_{1}} P_{2}^{t_{2}}$ where $P_{1}, P_{2}$ are distinct prime ideals of $R$ and $t_{i} \geq 1$ for all $i \in\{1,2\}$, then the following statements are equivalent.

(i): $I$ is a $S_{r}$-primary ideal of $R$.

(ii): $I$ is a weakly $S_{r}$-primary ideal of $R$.

(iii): There is exactly one $i \in\{1,2\}$ such that $r \in P_{i}$.

(3) If $I=P_{1}^{t_{1}} P_{2}^{t_{2}} \cdots P_{k}^{t_{k}}$ where $k \geq 3, P_{i}$ 's are distinct prime ideals of $R$ and $t_{i} \geq 1$ for all $i \in\{1, \ldots, k\}$, then $I$ is not a (weakly) $S_{r}$-primary ideal of $R$. 
Proof. Write $I=P_{1}^{t_{1}} P_{2}^{t_{2}} \ldots P_{k}^{t_{k}}$ where $P_{1}, P_{2}, \ldots, P_{k}$ are distinct prime ideals of $R$. First, we show that $I \cap S_{r}=\phi$ if and only if $r \notin \cap_{i=1}^{k} P_{i}$. Let $a \in I \cap S_{r}$. Since $I$ is proper, $a \neq 1$, and $a=r^{n} \in I$ for some $n \in \mathbb{N}$. Since $I \subseteq \cap_{i=1}^{k} P_{i}$, we have $r \in P_{i}$ for each $i \in\{1, \ldots, k\}$; i.e. $r \in \cap_{i=1}^{k} P_{i}$. Conversely, if $r \in \cap_{i=1}^{k} P_{i}$, then clearly $r^{t_{1}+\cdots+t_{k}} \in I \cap S_{r}$.

(1). Clear as $I$ is primary and $I \cap S_{r}=\phi$.

(2). Since $I \cap S_{r}=\phi$, then either $r \notin P_{i}$ for $i=1,2$ or $r \in P_{i}$ for exactly one $i \in\{1,2\}$.

(i) $\Rightarrow$ (ii). is straightforward.

(ii) $\Rightarrow$ (iii). Suppose $I$ is weakly $S$-primary and $r \notin P_{i}$ for all $i=1,2$. Since $I \neq\{0\}$, there is $0 \neq x \in I$, say $x:=a^{t_{1}} b^{t_{2}} \in I$ for some $a \in P_{1} \backslash P_{2}$ and $b \in P_{2} \backslash P_{1}$. Hence, $0 \neq a^{t_{1}} b^{t_{2}} \in I$. If $s a^{t_{1}} \in P_{1} P_{2} \subseteq P_{2}$ for some $s \in S_{r}$, then $s \in P_{2}$ which implies $r \in P_{2}$, a contradiction. Similarly, if $s b^{t_{2}} \in I \subseteq P_{1}$ for some $s \in S_{r}$, we get $r \in P_{1}$, a contradiction. Thus, $s a^{t_{1}} \notin P_{1} P_{2}=\sqrt{I}$ and $s b^{t_{2}} \notin I$ for all $s \in S_{r}$ and so $I$ is not a weakly $S_{r}$-primary ideal of $R$, a contradiction.

(iii) $\Rightarrow$ (i). Suppose, say, $r \in P_{1}$ and $r \notin P_{2}$ and choose $s=r^{t_{1}} \in S_{r}$. Let $a, b \in R$ such that $a b \in I$. If $a \in P_{2}$, then $s a \in P_{1} P_{2}=\sqrt{I}$. If $a \notin P_{2}$, then since $P_{2}^{t_{2}}$ is a primary ideal with radical $P_{2}$ and $a b \in I \subseteq P_{2}^{t_{2}}$, we conclude that $b \in P_{2}^{t_{2}}$. Thus, $s b \in I$. Therefore, $I$ is a $S_{r}$-primary ideal of $R$.

(3). Since $I \cap S_{r}=\phi$, we may assume without loss of generality that $r \in$ $\cap_{i=1}^{k-1} P_{i}$ and $r \notin P_{k}$. Choose $a_{i} \in P_{i}$ such that $a_{i} \notin P_{j}$ for all $i \neq j$. Then $\left(a_{1}^{t_{1}} a_{2}^{t_{2}} \cdots a_{k-1}^{t_{k-1}}\right) a_{k}^{t_{k}} \in I$ but $s\left(a_{1}^{t_{1}} a_{2}^{t_{2}} \cdots a_{k-1}^{t_{k-1}}\right) \notin P_{1} P_{2} \cdots P_{k}=\sqrt{I}$ and $s a_{k}^{t_{k}} \notin I$ for all $s \in S_{r}$. Hence, $I$ is not $S_{r}$-primary. Also, if $r \notin P_{i}$ for all $i$, then by a similar way, we conclude that $I$ is not $S_{r}$-primary.

Let $n \in \mathbb{N}$. For any integer $m \leq n$. In view of Theorem 1 , we determine all (weakly) $S_{m}$-primary ideals of the $\operatorname{ring} \mathbb{Z}_{n}($ resp. $\mathbb{Z})$ for any integer $m \leq n$.

Proposition 1. Let $n, m \in \mathbb{N}, m \leq n$ (resp. $m \in \mathbb{N}$ ) and $I$ be an ideal of $\mathbb{Z}_{n}$ (resp. $\mathbb{Z})$ disjoint with $S_{m}$. We have three cases:

(1) If $I=\left\langle p^{l}\right\rangle$ for some prime $p$ with $\operatorname{gcd}(p, m)=1$, then $I$ is (weakly) $S_{m^{-}}$ primary in $\mathbb{Z}_{n}$ (resp. in $\left.\mathbb{Z}\right)$.

(2) If $I=\left\langle p_{1}^{t_{1}} p_{2}^{t_{2}}\right\rangle$ where $p_{1}, p_{2}$ are distinct primes and $t_{i} \geq 1$ for all $i$, then $I$ is $S_{m}$-primary iff $I$ is weakly $S_{m}$-primary iff there is exactly one $i \in\{1,2\}$ such that $p_{i} \mid m$.

(3) If $I=\left\langle p_{1}^{t_{1}} p_{2}^{t_{2}} \cdots p_{k}^{t_{k}}\right\rangle$ where $k \geq 3, p_{i}$ 's are distinct primes and $t_{i} \geq 1$ for all $i$, then $I$ is not $S_{m}$-primary in $\mathbb{Z}_{n}$ (resp. in $\mathbb{Z}$ ). Moreover, $I$ is weakly $S_{m}$-primary iff $I=0$.

Recall that a ring $R$ is called a von Neumann regular ring if for each $a \in A$, there exists $b \in A$ such that $a=a^{2} b$ [16]. It is known from [10, Exercise 16, page 111] that a $\operatorname{ring} R$ is von Neumann regular if and only if $R$ is reduced and $\operatorname{dim} R=0$. Let $R$ be a ring, $M$ be an $R$-module. Recall that a submodule $N$ of $M$ is said to be pure in if $I N=N \cap I M$ for each ideal $I$ of $R$. A pure ideal of $R$ is just a pure submodule of the $R$-module $R$. The following proposition gives a condition for a weakly $S$-primary ideal to be an $S$-primary ideal.

Proposition 2. Let $S$ be a multiplicatively closed subset of a ring $R$ and $I$ be a weakly $S$-primary ideal of $R$.

(1) If $I^{2} \neq 0$, then $I$ is an $S$-primary ideal of $R$. 
(2) If $I$ is not an $S$-primary ideal, then $\sqrt{I}=\sqrt{0}$.

(3) If $R$ is a reduced ring (in particular, if $R$ is a von-Neumann regular ring), then either $I=\{0\}$ or $I$ is an $S$-primary ideal of $R$.

(4) If $I$ is pure that is not an $S$-primary ideal, then $I=0$.

Proof. (1) Suppose $a b \in I$ for some $a, b \in R$ and let $s$ be a weakly $S$-primary element of $I$. If $a b \neq 0$, then there is nothing to prove. So, assume that $a b=0$. In case of $a I \neq 0$, then $a x \neq 0$ for some $x \in I$. This implies $0 \neq a(x+b) \in I$ which implies either $s a \in \sqrt{I}$ or $s(x+b) \in I$ and so $s a \in \sqrt{I}$ or $s b \in I$. Assume that $b I \neq 0$. Then $b y \neq 0$ for some $y \in I$ and so $0 \neq(y+a) b \in I$. It follows that either $s(y+a) \in \sqrt{I}$ or $s b \in I$ and so again $s a \in \sqrt{I}$ or $s b \in I$. Now, assume that $a I=b I=0$. Since $I^{2} \neq 0$, there exist $c, d \in I$ with $c d \neq 0$. Hence, $0 \neq c d=(a+c)(b+d) \in I$ which implies $s(a+c) \in \sqrt{I}$ or $s(b+d) \in I$. Therefore, $s a \in \sqrt{I}$ or $s b \in I$ and $I$ is an $S$-primary ideal of $R$.

(2) and (3) are follow clearly by (1).

(4) Since $I$ not an $S$-primary ideal and it is pure, we conclude $I=I^{2}=0$ by (1).

In the following, we conclude a result which is similar to Nakayama's Lemma.

Corollary 1. Let $S$ be a multiplicatively closed subset of a ring $R$ and $M$ be an $R$-module. If $I M=M$ for a weakly $S$-primary ideal $I$ of $R$ that is not $S$-primary, then $M=0$.

Proof. Since $I$ is a weakly $S$-primary ideal of $R$ that is not $S$-primary, we have $I^{2}=0$. Thus $M=I M=I^{2} M=0$.

Proposition 3. Let $S$ be a multiplicatively closed subset of a ring $R$ and $I$ a non-zero ideal of $R$ disjoint with $S$. If $I$ is a weakly $S$-n-ideal, then $s I \subseteq \sqrt{0}$ for some $s \in S$. Furthermore, if, $S \subseteq \operatorname{reg}(R)$, then $I \subseteq \sqrt{0}$.

Proof. Let $s$ be a weakly $S$-n-element of $I$. Let $0 \neq a \in I$. Then $a \cdot 1 \in I$ implies $s a \in \sqrt{0}$ or $s=s \cdot 1 \in I$. But $s \notin I$ as $I \cap S=\emptyset$. Thus, $s I \subseteq \sqrt{0}$. Furthermore, if $S \subseteq \operatorname{reg}(R)$, then clearly $I \subseteq \sqrt{0}$

Next, we present some equivalent statements to characterize weakly $S$-primary ideals of a ring $R$.

Theorem 2. Let $S$ be a multiplicatively closed subset of a ring $R$ and $I$ be an ideal of $R$ disjoint with $S$. Then the following assertions are equivalent.

(1) $I$ is a weakly $S$-primary ideal of $R$.

(2) There exists $s \in S$ such that $(I: a)=(0: a)$ or $(I: a) \subseteq(I: s)$ for each $a \notin(\sqrt{I}: s)$.

(3) There exists $s \in S$ such that for any $a \in R$ and for any ideal $K$ of $R$, if $0 \neq a K \subseteq I$, then $s a \subseteq \sqrt{I}$ or $s K \subseteq I$.

(4) There exists $s \in S$ such that for any two ideals $J, K$ of $R$, if $0 \neq J K \subseteq I$, then $s J \subseteq \sqrt{I}$ or $s K \subseteq I$.

Proof. $(1) \Rightarrow(2)$. Suppose that $s$ is a weakly $S$-primary element of $I$ and choose $a \in R \backslash(\sqrt{I}: s)$. Assume that $(I: a) \neq(0: a)$. Then $0 \neq a b \in I$ for some $b \in R$. Since $s a \notin \sqrt{I}$, we have $s b \in I$. Now let $x \in(I: a)$. If $x a \neq 0$, then since $a \notin(\sqrt{I}: s)$, we have $s x \in I$ and so $x \in(I: s)$. If $x a=0$, then we have 
$0 \neq a b=a(b+x) \in I$ and $s a \notin \sqrt{I}$ implies $s(b+x) \in I$. Thus, $s x \in I$ and $x \in(I: s)$ as needed.

$(2) \Rightarrow(3)$. Choose $s \in S$ as in (2) and assume that for $a \in R$ and an ideal $K$ of $R, 0 \neq a K \subseteq I$ and $s a \nsubseteq \sqrt{I}$. Then $a \notin(\sqrt{I}: s)$ and $K \subseteq(I: a) \backslash(0: a)$ imply $K \subseteq(I: s)$ by (2). Thus $s K \subseteq I$ as required.

$(3) \Rightarrow(4)$. Choose $s \in S$ as in (3) and suppose that $0 \neq J K \subseteq I$ and $s J \nsubseteq \sqrt{I}$ for some ideals $J, K$ of $R$. Then there exists $a \in J$ with $s a \notin \sqrt{I}$. If $a K \neq 0$, then by (3), we have $s K \subseteq I$. Assume that $a K=0$. Since $J K \neq 0$, we can write $b K \neq 0$ for some $b \in J$. If $s b \notin \sqrt{I}$, then $s K \subseteq I$ again by (3). Now suppose that $s b \in \sqrt{I}$. Since $s a \notin \sqrt{I}$, we have $s(a+b) \notin \sqrt{I}$. Hence, $0 \neq(a+b) K \subseteq I$ implies $s K \subseteq I$ and we are done.

$(4) \Rightarrow(1)$. Choose $s \in S$ as in (4) and let $a, b \in R$ with $0 \neq a b \in I$. Put $J=\langle a\rangle$ and $K=\langle b>$ in (4), so we get the claim.

Proposition 4. Let $S$ be a multiplicatively closed subset of a ring $R$ and $I$ be an ideal of $R$ disjoint with $S$.

(1) If $(I: s)$ is a weakly primary ideal of $R$ for some $s \in S$, then $I$ is a weakly $S$-primary ideal of $R$.

(2) If $S \subseteq \operatorname{reg}(R)$ and $I$ is a non-zero weakly $S$-primary ideal of $R$, then $(I: s)$ is a weakly primary ideal of $R$ for every weakly $S$-primary element $s$ of $I$.

Proof. (1). Suppose for some $s \in S$ that $(I: s)$ is a weakly primary ideal of $R$ and let $a, b \in R$ such that $0 \neq a b \in I \subseteq(I: s)$. Then either $a \in \sqrt{(I: s)}$ or $b \in(I: s)$ and so either $s a \in \sqrt{I}$ or $s b \in I$. Therefore, $s$ is a weakly $S$-primary element of $I$ and we are done.

(2). Suppose $I$ is weakly $S$-primary and let $s$ be a weakly $S$-primary element of $I$. Let $a, b \in R$ such that $0 \neq a b \in(I: s)$ so that $a b s \in I$. If $a b s=0$, then $a b=0$ since $S \subseteq \operatorname{reg}(R)$, a contradiction. Thus, $a b s \neq 0$ and so by assumption, either $s a \in \sqrt{I}$ or $s^{2} b \in I$. Suppose $s^{2} b \in I$. If $s^{2} b=0$, then $S \subseteq \operatorname{reg}(R)$ implies $a b=b=0$, a contradiction. Thus, $s^{2} b \neq 0$ and since $s^{3} \notin \sqrt{I}$, then $s b \in I$ and $b \in(I: s)$. Suppose $s a \in \sqrt{I}$, say, $s^{n} a^{n} \in I$ for some $n \in \mathbb{N}$. If $s^{n} a^{n}=0$, then $a^{n}=0$ as $S \subseteq \operatorname{reg}(R)$ and so $a \in \sqrt{0} \subseteq \sqrt{(I: s)}$. Otherwise, if $s^{n} a^{n} \neq 0$, then $s a^{n} \in I$ since $s^{n+1} \notin \sqrt{I}$. It follows again that $a \in \sqrt{(I: s)}$ and hence $(I: s)$ is a weakly primary ideal of $R$.

The assumption " $I$ is non-zero" is necessary in Proposition 4 (2). For example, consider $R=\mathbb{Z}_{24}$ and $S=\left\{2^{n}: n \in \mathbb{N}\right\}$. Observe that $I=\{0\}$ is always a weakly $S$-primary ideal of $\mathbb{Z}_{24}$ but $(I: 4)=<\overline{6}>$ is not weakly primary.

Remark 1. Let $S_{1} \subseteq S_{2}$ be two multiplicatively closed subsets of a ring $R$. Then it is clear that any weakly $S_{1}$-primary ideal $I$ of $R$ with $I \cap S_{2}=\emptyset$ is a weakly $S_{2}$ primary ideal of $R$. However, the converse does not necessarily hold: For example, consider the multiplicatively closed subsets $S_{1}=\{1\}$ and $S_{2}=\left\{2^{n}: n \in \mathbb{N}\right\}$ of $R=\mathbb{Z}[X]$. In Example 1 (1), it is shown that $I=8 X \mathbb{Z}[X]$ is a weakly $S_{2}$-primary ideal which is not a weakly $S_{1}$-primary.

Let $S$ be a multiplicatively closed subset of a ring $R$. The saturation of $S$ is the set $S^{*}=\{x \in R: x y \in S$ for some $y \in R\}$, see [10]. It is clear that $S^{*}$ is a multiplicatively closed subset of $R$ and that $S \subseteq S^{*}$. 
Proposition 5. Let $S$ be a multiplicatively closed subset of a ring $R$ and $I$ be an ideal of $R$ disjoint with $S$. Then $I$ is a weakly $S$-primary ideal of $R$ if and only if $I$ is a weakly $S^{*}$-primary ideal of $R$.

Proof. Suppose $I$ is a weakly $S^{*}$-primary ideal of $R$ associated to a weakly $S$ primary element $s^{*}$ of $I$. Let $a, b \in R$ such that $0 \neq a b \in I$. Then $s^{*} a \in \sqrt{I}$ or $s^{*} b \in I$. Choose $y \in R$ such that $s=s^{*} y \in S$. Then $s a \in \sqrt{I}$ or $s b \in I$ as desired. The converse is clear.

Proposition 6. Let $S$ be a multiplicatively closed subset of a ring $R$. If $I$ is a weakly $S$-primary ideal of $R$ and $\{0\}$ is an $S$-primary ideal, then $\sqrt{I}$ is an $S$-prime ideal of $R$.

Proof. Let $s_{1} \in S$ be a weakly $S$-primary element of $I$ and suppose $\{0\}$ is an $S$ primary ideal associated with $s_{2} \in S$. Since $I \cap S=\emptyset$, we have $\sqrt{I} \cap S=\emptyset$. Let $a, b \in R$ with $a b \in \sqrt{I}$. Then $a^{n} b^{n} \in I$ for some positive integer $n$. If $a^{n} b^{n} \neq 0$, then we have either $s_{1} a^{n} \in \sqrt{I}$ or $s_{1} b^{n} \in I$; i.e. $s_{1} a \in \sqrt{I}$ or $s_{1} b \in \sqrt{I}$. Now, suppose that $a^{n} b^{n}=0$. This yields either $s_{2} a^{n} \in \sqrt{0}$ or $s_{2} b^{n}=0$ and so $s_{2} a \in \sqrt{0} \subseteq \sqrt{I}$ or $s_{2} b \in \sqrt{0} \subseteq \sqrt{I}$. Thus, $\sqrt{I}$ is an $S$-prime ideal of $R$ associated with $s=s_{1} s_{2}$.

Note that the condition " $\{0\}$ is an $S$-primary" in Proposition 6 is irreplaceable. For example the zero ideal in $\mathbb{Z}_{12}$ is a weakly $\{1\}$-primary that is not $\{1\}$-primary and $\sqrt{0}=\langle\overline{6}\rangle$ is not $\{1\}$-prime.

Proposition 7. Let $S$ be a multiplicatively closed subset of a ring $R$ and $I$ be a weakly $S$-primary ideal of $R$. For any ideal $J$ of $R$ with $J \cap S \neq \emptyset, I \cap J$ and $I J$ are weakly $S$-primary ideals of $R$.

Proof. Since $I \cap S=\emptyset$, clearly we have $(I \cap J) \cap S=I J \cap S=\emptyset$. Let $s \in S$ be a weakly $S$-primary element of $I$ and let $0 \neq a b \in I \cap J$. Then $s a \in \sqrt{I}$ or $s b \in I$. Choose $t \in J \cap S$. Then $s t a \in \sqrt{I} \cap J \subseteq \sqrt{I} \cap \sqrt{J}=\sqrt{I \cap J}$ or $s t b \in I \cap J$. Thus $I \cap J$ is a weakly $S$-primary ideal of $R$. The rest of the proof is very similar.

We not in Proposition 7 , that if $S \neq\{1\}, J I$ need not be weakly primary even if $I$ is weakly primary. For example let $R=\mathbb{Z}_{12}, S=\{\overline{1}, \overline{3}, 9\}, J=\langle\overline{3}\rangle$ and $I=\langle\overline{2}\rangle$. Then $J I=\langle\overline{6}\rangle$ is not a weakly primary ideal of $R$.

Proposition 8. Let $S$ be a multiplicatively closed subset of a ring $R$ and $I_{1}, \ldots, I_{n}$ be weakly $S$-primary ideals of $R$. If $\sqrt{I_{i}}=\sqrt{I_{j}}$ for all $i, j=1, \ldots, n$, then $I=\bigcap_{i=1}^{n} I_{i}$ is a weakly $S$-primary ideal of $R$. In particular, $I$ is weakly $S$-primary if $I_{1}, \ldots, I_{n}$ are not $S$-primary.

Proof. Since for each $i=1, \ldots, n, I_{i} \cap S=\emptyset$, then we have $\left(\bigcap_{i=1}^{n} I_{i}\right) \cap S=\emptyset$. For each $i=1, \ldots, n$, let $s_{i} \in S$ be a weakly $S$-primary element of $I_{i}$. Put $s:=\prod_{i=1}^{n} s_{i} \in S$.

Suppose for $a, b \in R$ that $0 \neq a b \in I$ but $s b \notin I$. Then $s b \notin I_{j}$ for some $j=1, \ldots, n$. Since $s_{j}$ is a weakly $S$-primary element of $I_{j}$ and clearly $s_{j} b \notin I_{j}$, we have $s_{j} a \in$ $\sqrt{I_{j}}$. Since $\sqrt{I}=\bigcap_{i=1}^{n} \sqrt{I_{i}}=\sqrt{I_{i}}$, then $s a \in \sqrt{I}$ as required. In particular if all $I_{j}$ 's 
are not $S$-primary, then by Proposition $2(2)$, we have $\sqrt{I}=\bigcap_{i=1}^{n} \sqrt{I_{i}}=\sqrt{0}=\sqrt{I_{j}}$ for all $i=1, \ldots, n$ and the result follows similarly.

We note that the condition " $\sqrt{I_{i}}=\sqrt{I_{j}}$ for all $i, j=1, \ldots, n$ " in Proposition 8 can not be ignored. For example, in the ring $\mathbb{Z}_{30}$, the ideals $\langle\overline{2}\rangle$ and $\langle\overline{3}\rangle$ are (weakly) $S_{5}$-primary. But, $\langle\overline{2}\rangle \cap\langle\overline{3}\rangle=\langle\overline{6}\rangle$ is not (weakly) $S_{5}$-primary by (2) of Proposition 1. Also, we can see that the converse of Proposition 8 need not be true in general. For example the ideals $I=\langle\overline{12}\rangle$ and $J=\langle\overline{18}\rangle$ are not weakly $\{1\}$-primary in $\mathbb{Z}_{36}$. On the other hand $\sqrt{I}=\sqrt{J}=\langle\overline{6}\rangle$ and $I \cap J=\langle\overline{0}\rangle$ is weakly $\{1\}$-primary in $\mathbb{Z}_{36}$.

Let $S$ and $T$ be multiplicatively closed subsets of a ring $R$ with $S \subseteq T$. Then clearly, $T^{-1} S=\left\{\frac{s}{t}: t \in T, s \in S\right\}$ is a multiplicatively closed subset of $T^{-1} R$. Moreover, if $I$ is an ideal of $R$, then $I_{S}=\{x \in R: x s \in I$ for some $s \in S\}$.

Proposition 9. Let $S, T$ be multiplicatively closed subsets of a ring $R$ with $S \subseteq T$ and $I$ be an ideal of $R$ disjoint with $T$.

(1) If $I$ is a weakly $S$-primary ideal of $R$, then $T^{-1} I$ is a weakly $T^{-1} S$-primary ideal of $T^{-1} R$ and $T^{-1} I \cap R=(I: s) \cup 0_{T}$ for some $s \in S$.

(2) If $T \subseteq \operatorname{reg}(R)$, the converse of (1) is true.

Proof. (1) Suppose $I$ is a weakly $S$-primary ideal of $R$ and suppose $T^{-1} S \cap T^{-1} I \neq$ $\phi$, say, $\frac{a}{t} \in T^{-1} S \cap T^{-1} I$. Then clearly $a \in S$ and $t a \in I$ for some $t \in T$. Since $S \subseteq T$, then $t a \in T \cap I$, a contradiction. Thus, $T^{-1} I$ is proper in $T^{-1} R$ and $T^{-1} \bar{S} \cap T^{-1} I=\phi$. Let $s \in S$ be a weakly $S$-primary element of $I$ and choose $\frac{s}{1} \in T^{-1} S$. Suppose $a, b \in R$ and $t_{1}, t_{2} \in T$ with $0 \neq \frac{a}{t_{1}} \frac{b}{t_{2}} \in T^{-1} I$ and $\frac{s}{1} \frac{a}{t_{1}} \notin \sqrt{T^{-1} I}$. Then $0 \neq t a b \in I$ for some $t \in T$ and one can easily verify that $s a \notin \sqrt{I}$. Since $I$ is a weakly $S$-primary ideal, we must have $s t b \in I$. Thus, $\frac{s}{1} \frac{b}{t_{2}}=\frac{s t b}{t t_{2}} \in T^{-1} I$ as needed. Now, let $r \in T^{-1} I \cap R$ and choose $i \in I, t \in T$ such that $\frac{r}{1}=\frac{i}{t}$. Then $v r \in I$ for some $v \in T$. If $v r=0$, then $r \in 0_{T}$. If $v r \neq 0$, then as $I$ is weakly $S$-primary, we have either $s v \in \sqrt{I}$ or $s r \in I$. But $s v \notin \sqrt{I}$ as $T \cap \sqrt{I}=\phi$ and so $s r \in I$. It follows that $r \in(I: s)$ for some $S$-element $s$ of $I$. Since clearly $(I: s) \cup 0_{T} \subseteq T^{-1} I \cap R$ for all $s \in T$, the proof is completed.

(2) Suppose that $0 \neq a b \in I$ for $a, b \in R$ and let $\frac{s}{t}$ be a weakly $T^{-1} S$-primary element of $T^{-1} I$. Since $S \subseteq T \subseteq \operatorname{reg}(R)$, we have $0 \neq \frac{a}{1} \frac{b}{1} \in T^{-1} I$. Hence, $\frac{s}{t} \frac{a}{1} \in \sqrt{T^{-1} I}$ or $\frac{s}{t} \frac{b}{1} \in T^{-1} I$. Thus, there exists $n \in \mathbb{N}$ such that $u s^{n} a^{n} \in I$ or $v s b \in I$ for some $v, u \in T$. If $u s^{n} a^{n} \in I$, then $a^{n}=\frac{u s^{n} a^{n}}{s^{n} u} \in T^{-1} I \cap R=(I: s) \cup 0_{T}$. If $a^{n} \in 0_{T}$, then $a^{n}=0 \in(I: s)$ as $T \subseteq \operatorname{reg}(R)$. Hence, $s a \in \sqrt{I}$. Otherwise, if $v s b \in I$, then similarly, we get $s b \in I$. Therefore, $I$ is a weakly $S$-primary ideal of $R$.

In particular, if $S=T$, then all elements of $T^{-1} S$ are units in $T^{-1} R$. As a special case of of Proposition 9, we have the following.

Corollary 2. Let $S$ be a multiplicatively closed subset of a ring $R$ and $I$ be an ideal of $R$ disjoint with $S$. If $I$ is a weakly $S$-primary ideal of $R$, then $S^{-1} I$ is a weakly primary ideal of $S^{-1} R$ and $S^{-1} I \cap R=(I: s) \cup 0_{S}$ for some $s \in S$. The converse holds if $S \subseteq \operatorname{reg}(R)$.

Proof. Suppose $I$ is a weakly $S$-primary ideal. Then $S^{-1} I$ is a weakly $S^{-1} S$-primary ideal of $S^{-1} R$ by Proposition 9. Let $a, b \in R, s_{1}, s_{2} \in S$ with $0 \neq \frac{a}{s_{1}} \frac{b}{s_{2}} \in S^{-1} I$. 
Then by assumption, $\frac{s}{t} \frac{a}{s_{1}} \in \sqrt{S^{-1} I}$ or $\frac{s}{t} \frac{b}{s_{2}} \in S^{-1} I$ for some $S^{-1} S$-element $\frac{s}{t}$ of $S^{-1} I$. Since $\frac{s}{t}$ is a unit in $S^{-1} R$, then $S^{-1} I$ is a weakly primary ideal of $S^{-1} R$ as required.

The proof of the other part is similar to that of (1) of Proposition 9. Conversely let $0 \neq a b \in I$ for $a, b \in R$ and choose $s \in S$ such that $S^{-1} I \cap R=(I: s) \cup 0_{S}$. Since $S \subseteq \operatorname{reg}(R)$, we have $0 \neq \frac{a}{1} \frac{b}{1} \in S^{-1} I$. Hence, $\frac{a}{1} \in \sqrt{S^{-1} I}=S^{-1} \sqrt{I}$ or $\frac{b}{1} \in S^{-1} I$. Thus, $u a^{n} \in I$ for some $n \in \mathbb{N}$ or $v b \in I$ for some $u, v \in S$. If $u a^{n} \in I$, then $a^{n}=\frac{u a^{n}}{u} \in S^{-1} I \cap R=(I: s) \cup 0_{T}$ and so it can be easily checked that $s a \in \sqrt{I}$. Similarly, if $v b \in I$, then $s b \in I$ and the result follows.

Corollary 3. Let $S \subseteq \operatorname{reg}(R)$ be a multiplicatively closed subset of a ring $R$. If every ideal in $S^{-1} R$ is radical, then every weakly $S$-primary ideal of $R$ is weakly S-prime.

Proof. Let $I$ be a weakly $S$-primary ideal of R. Then $S^{-1} I$ is weakly primary in $S^{-1} R$ by Corollary 2 and so $S^{-1} I$ is weakly prime as $S^{-1} I$ is a radical ideal of $S^{-1} R$. Hence, $S^{-1} I \cap R$ is weakly prime in $R$. Indeed, if $0 \neq a b \in S^{-1} I \cap R$ for $a, b \in R$, then $0 \neq \frac{a}{1} \frac{b}{1} \in S^{-1} I$ since $S \subseteq \operatorname{reg}(R)$. It follows that $\frac{a}{1} \in S^{-1} I$ or $\frac{b}{1} \in S^{-1} I$ and so $a \in S^{-1} I \cap R$ or $b \in S^{-1} I \cap R$. Therefore, by Corollary 2 $(I: s)=S^{-1} I \cap R$ is weakly prime in $R$. Thus, $I$ is a weakly $S$-prime ideal of $R$ by $[1$, Proposition 14].

Next, we characterize rings in which the zero ideal is the only weakly $S$-primary ideal.

Theorem 3. Let $S$ be a multiplicatively closed subset of a ring $R$. The following are equivalent.

(1) $\langle 0\rangle$ is the only weakly $S$-primary ideal of $R$.

(2) $\langle 0\rangle$ is the only weakly $S$-prime ideal of $R$.

(3) $R$ is a domain and $S^{-1} R$ is a field.

(4) $S \subseteq \operatorname{reg}(R)$ and $S^{-1} R$ is a field.

Proof. $(1) \Rightarrow(2)$. Clear.

$(2) \Rightarrow(3) . \quad[1$, Proposition 15$]$.

$(3) \Rightarrow(4)$. Clear.

$(4) \Rightarrow(1)$. Suppose $I$ is a non-zero weakly $S$-primary ideal in $R$ and let $0 \neq r \in I$. Then $0 \neq \frac{r}{1} \in S^{-1} I$ and so there is $0 \neq \frac{a}{s} \in S^{-1} R$ such that $\frac{r a}{s}=\frac{1}{1}$. Thus, $u(r a-s)=0$ for some $s \in S$. Since $S \subseteq \operatorname{reg}(R)$, then $r a=s \in I \cap S$, a contradiction.

Proposition 10. Let $f: R_{1} \rightarrow R_{2}$ be a ring homomorphism and $S$ be a multiplicatively closed subset of $R_{1}$. Then the following statements hold.

(1) If $f$ is an epimorphism and $I$ is a weakly $S$-primary ideal of $R_{1}$ containing $\operatorname{Ker}(f)$, then $f(I)$ is a weakly $f(S)$-primary ideal of $R_{2}$.

(2) If $f$ is a monomorphism and $J$ is an $f(S)$-primary ideal of $R_{2}$, then $f^{-1}(J)$ is a weakly $S$-primary ideal of $R_{1}$.

Proof. (1) First, we show that $f(I) \cap f(S)=\emptyset$. Assume not. Then there exists $u \in f(I) \cap f(S)$ which implies $u=f(x)=f(s)$ for some $x \in I$ and $s \in S$. Hence, $x-s \in \operatorname{Ker}(f) \subseteq I$ and $s \in I \cap S$ which is a contradiction. Suppose $0 \neq a b \in f(I)$ for some $a, b \in R_{2}$, say $a:=f(x)$ and $b:=f(y)$ for some $x, y \in R_{1}$. 
Then $0 \neq f(x) f(y) \in f(I)$ and clearly we have $0 \neq x y \in I$. If we choose a weakly $S$-element $s$ of $I$, then either $s x \in \sqrt{I}$ or $s y \in I$. Thus, clearly we have $f(s) a \in f(\sqrt{I})=\sqrt{f(I)}$ or $f(s) b \in f(I)$ and so $f(I)$ is a weakly $f(S)$-primary ideal of $R_{2}$.

(2) Suppose $0 \neq a b \in f^{-1}(J)$ for some $a, b \in R_{1}$. Then $0 \neq f(a b)=f(a) f(b) \in J$. Since $J$ is an $f(S)$-primary ideal of $R_{2}$, there exists a weakly $f(S)$-element $f(s)$ of $J$ such that $f(s) f(a) \in \sqrt{J}$ or $f(s) f(b) \in J$. Thus, $s a \in f^{-1}(\sqrt{J})=\sqrt{f^{-1}(J)}$ or $s b \in f^{-1}(J)$.

Let $S$ be a multiplicatively closed subset of a ring $R$ and $I$ be an ideal of $R$ disjoint with $S$. If we denote $r+I \in R / I$ by $\bar{r}$, then clearly the set $\bar{S}=\{\bar{s}: s \in S\}$ is a multiplicatively closed subset of $R / I$. In view of Proposition 10, we conclude the following result for weakly $\bar{S}$-primary ideals of $R / I$.

Corollary 4. Let $S$ be a multiplicatively closed subset of a ring $R$ and $I, J$ are two ideals of $R$ with $I \subseteq J$.

(1) If $J$ is a weakly $S$-primary ideal of $R$, then $J / I$ is a weakly $\bar{S}$-primary ideal of $R / I$.

(2) If $R$ is a subring of $R^{\prime}$ and $I^{\prime}$ is a weakly $S$-primary ideal of $R^{\prime}$, then $I^{\prime} \cap R$ is a weakly $S$-primary ideal of $R$.

(3) If $J / I$ is a weakly $\bar{S}$-primary ideal of $R / I$ and $I$ is a $S$-primary ideal of $R$, then $J$ is a $S$-primary ideal of $R$.

(4) If $J / I$ is a weakly $\bar{S}$-primary ideal of $R / I$ and $I$ is a weakly $S$-primary ideal of $R$, then $J$ is a weakly $S$-primary ideal of $R$.

Proof. (1) Note that $(J / I) \cap \bar{S}=\phi$ if and only if $J \cap S=\phi$. The claim follows from (1) of Proposition 10 with the canonical epimorphism $\pi: R \rightarrow R / I$ defined by $\pi(r)=r+I$ for all $r \in R$.

(2) Consider the natural injection $i: R \rightarrow R^{\prime}$ defined by $i(r)=r$ for all $r \in R$. Since $I^{\prime}$ is a weakly $S$-primary ideal of $R^{\prime}$, by Proposition $10(2), i^{-1}\left(I^{\prime}\right)=I^{\prime} \cap R$ is a weakly $S$-primary ideal of $R$.

(3) Let $\overline{s_{1}}=s_{1}+I \in \bar{S}$ be a weakly $\bar{S}$-primary element of $J / I$ and suppose $I$ is an $S$-primary ideal of $R$ associated with $s_{2} \in S$. Let $a b \in J$ for some $a, b \in R$. If $a b \in I$, then $s_{2} a \in \sqrt{I} \subseteq \sqrt{J}$ or $s_{2} b \in I \subseteq J$. If $a b \notin I$, then $I \neq(a+I)(b+I) \in J / I$ which yields $\left(s_{1}+I\right)(a+I) \in \sqrt{J / I}=\sqrt{J} / I$ or $\left(s_{1}+I\right)(b+I) \in J / I$. Thus $s_{1} a \in \sqrt{J}$ or $s_{1} b \in J$. Now, put $s=s_{1} s_{2} \in S$. Then $J$ is an $S$-primary ideal of $R$ associated with $s$.

(4) is similar to $(3)$.

Proposition 11. Let $S$ be a multiplicatively closed subset of a ring $R$ and $I, J$ be two weakly $S$-primary ideals of $R$ such that $(I+J) \cap S=\emptyset$. Then $I+J$ is a weakly $S$-primary ideal of $R$.

Proof. Set $\bar{S}_{1}=\{s+(I \cap J): s \in S\}$ and $\bar{S}=\{s+J: s \in S\}$ which are both multiplicatively closed subsets of rings $R /(I \cap J)$ and $R / J$, respectively. Here, $I /(I \cap J)$ is a weakly $\bar{S}_{1}$-primary ideal of $R /(I \cap J)$ by Corollary 4 (1). Since $\bar{S}_{1} \subseteq \bar{S}$, then $I /(I \cap J)$ is also a weakly $\bar{S}$-primary ideal of $R /(I \cap J)$ by Remark 1 . Thus, $(I+J) / J$ is a weakly $\bar{S}$-primary ideal of $R / J$ by the isomorphism $(I+J) / J \cong$ $I /(I \cap J)$. It follows from Corollary $4(4)$ that $I+J$ is a weakly $S$-primary ideal of $R$. 
Theorem 4. Let $S_{1}, S_{2}$ be multiplicatively closed subsets of rings $R_{1}, R_{2}$ respectively and $I_{1}, I_{2}$ be non-zero ideals of $R_{1}, R_{2}$, respectively. Let $R=R_{1} \times R_{2}$, $S=S_{1} \times S_{2}$ and $I=I_{1} \times I_{2}$. Then the following statements are equivalent.

(1) $I$ is a weakly $S$-primary ideal of $R$.

(2) $I_{1}$ is an $S_{1}$-primary ideal of $R_{1}$ and $S_{2} \cap I_{2} \neq \emptyset$ or $I_{2}$ is a $S_{2}$-primary ideal of $R_{2}$ and $S_{1} \cap I_{1} \neq \emptyset$.

(3) $I$ is a $S$-primary ideal of $R$.

Proof. (1) $\Rightarrow(2)$. Let $s=\left(s_{1}, s_{2}\right)$ be a weakly $S$-primary element of $I$. Assume on the contrary that $S_{1} \cap I_{1}=S_{2} \cap I_{2}=\emptyset$. Since $I_{1}$ and $I_{2}$ are non-zero, choose a non-zero element $(a, b) \in I$. Then $\left(0_{R_{1}}, 0_{R_{2}}\right) \neq(a, 1)(1, b) \in I$ which yields either $s(a, 1) \in \sqrt{I}$ or $s(1, b) \in I$. Thus clearly we have either $s_{2}^{n} \in S_{2} \cap I_{2}$ for some positive integer $n$ or $s_{1} \in S_{1} \cap I_{1}$, a contradiction. Without loss of generality, we may assume that $S_{1} \cap I_{1} \neq \emptyset$ and we will prove that $I_{2}$ is a $S_{2}$-primary ideal of $R_{2}$. First, we note that $S_{2} \cap I_{2}=\emptyset$ as $S \cap I=\emptyset$. Suppose $a b \in I_{2}$ for some $a, b \in R_{2}$. Choose $0_{R_{1}} \neq u \in S_{1} \cap I_{1}$. Hence $\left(0_{R_{1}}, 0_{R_{2}}\right) \neq(u, a)(1, b) \in I$ implies either $s(u, a) \in \sqrt{I}$ or $s(1, b) \in I$. Therefore, $s_{2} a \in \sqrt{I_{2}}$ or $s_{2} b \in I_{2}$, as needed.

$(2) \Rightarrow(3)$. We may assume that $I_{1}$ is a $S_{1}$-primary ideal of $R_{1}$ with an $S_{1}$-primary element $s_{1} \in S_{1}$ and $S_{2} \cap I_{2} \neq \emptyset$. Choose $s_{2} \in S_{2} \cap I_{2}$. Let $\left(a_{1}, a_{2}\right)\left(b_{1}, b_{2}\right) \in I$ for some $a_{1}, b_{1} \in R_{1}$ and $a_{2}, b_{2} \in R_{2}$. Then $a_{1} b_{1} \in I_{1}$ which implies $s_{1} a_{1} \in \sqrt{I_{1}}$ or $s_{1} b_{1} \in I_{1}$. Now set $s=\left(s_{1}, s_{2}\right) \in S$. Observe that $s\left(a_{1}, a_{2}\right) \in \sqrt{I}$ or $s\left(b_{1}, b_{2}\right) \in I$ and thus $I$ is a $S$-primary ideal of $R$.

$(3) \Rightarrow(1)$. is straightforward.

Let $R_{1}$ and $R_{2}$ be two rings. Observe that if $I_{1}$ is a weakly $S_{1}$-primary ideal of $R_{1}$ and $I_{2}$ is a weakly $S_{2}$-primary ideal of $R_{2}$, then $I_{1} \times I_{2}$ need not to be a weakly $S_{1} \times S_{2}$-primary ideal of $R_{1} \times R_{2}$. To see this, consider $R_{1}=R_{2}=\mathbb{Z}$, the multiplicatively closed subsets $S_{1}=\mathbb{Z} \backslash 3 \mathbb{Z}$ and $S_{2}=\mathbb{Z} \backslash 2 \mathbb{Z}$ and the ideals $I_{1}=9 \mathbb{Z}$ and $I_{2}=4 \mathbb{Z}$ of $R_{1}, R_{2}$, respectively. It is clear that $I_{1}$ is a weakly $S_{1}$-primary ideal of $R_{1}$ and $I_{2}$ is a weakly $S_{2}$-primary ideal of $R_{2}$. However, $I=I_{1} \times I_{2}$ is not a weakly $S_{1} \times S_{2}$-primary ideal of $R_{1} \times R_{2}$ as $(0,0) \neq(3,4)(3,1) \in I$ but for each $\left(s_{1}, s_{2}\right) \in S_{1} \times S_{2}$, neither $\left(s_{1}, s_{2}\right)(3,4) \in I$ nor $\left(s_{1}, s_{2}\right)(3,1) \in \sqrt{I}=3 \mathbb{Z} \times 2 \mathbb{Z}$.

Theorem 5. Let $R=R_{1} \times R_{2} \times \cdots \times R_{n}, S=S_{1} \times S_{2} \times \cdots \times S_{n}$ where $R_{i}$ 's are rings and $S_{i}$ is a multiplicatively closed subset of $R_{i}$ for each $i=1,2, \ldots, n$. For an ideal $I=I_{1} \times I_{2} \times \cdots \times I_{n}$, the following are equivalent.

(1) $I$ is a weakly $S$-primary ideal of $R$.

(2) For some $i=1,2, \ldots, n, I_{i}$ is an $S$-primary ideal of $R_{i}$ and $S_{j} \cap I_{j} \neq \emptyset$ for all $j \neq i$.

Proof. We prove the claim by using mathematical induction on $n$. It is obvious for $n=1$. Also the claim follows from Theorem 4 for $n=2$. Now, assume that the claim holds for all $k<n$, then we will prove for $k=n$. Let $I=I_{1} \times I_{2} \times \cdots \times I_{n}$ be a weakly $S$-primary ideal of $R$. Then Theorem 4 implies that $I=I^{\prime} \times I_{n}$ where $I^{\prime}=I_{1} \times I_{2} \times \cdots \times I_{n-1}$ is a weakly $S$-primary ideal of $R^{\prime}=R_{1} \times R_{2} \times \cdots \times R_{n-1}$ and $S_{n} \cap I_{n} \neq \emptyset$. Thus we are done from the induction hypothesis.

\section{Weakly $S$-primary ideals of Idealization and Amalgamation Rings}

Let $R$ be a ring and let $M$ be an $R$-module. We recall that $R(+) M=\{(r, m): r \in R, m \in M\}$ with coordinate-wise addition and multiplication defined as $\left(r_{1}, m_{1}\right)\left(r_{2}, m_{2}\right)=$ 
$\left(r_{1} r_{2}, r_{1} m_{2}+r_{2} m_{1}\right)$ is a commutative ring with identity $(1,0)$. This ring is called the idealization of the $R$-module $M$. For an ideal $I$ of $R$ and a submodule $N$ of $M, I(+) N$ is an ideal of $R(+) M$ if and only if $I M \subseteq N$. Moreover, the radical of $I(+) M$ is $\sqrt{I(+) M}=\sqrt{I}(+) M$. If $S$ is a multiplicatively closed subset of $R$, then clearly the sets $S(+) M=\{(s, m): s \in S, m \in M\}$ and $S(+) 0=\{(s, 0): s \in S\}$ are multiplicatively closed subsets of the ring $R(+) M$.

Next, we discuss the relation between weakly $S$-primary ideals of a $\operatorname{ring} R$ and those of the idealization $\operatorname{ring} R(+) M$.

Proposition 12. Let $S$ be a multiplicatively closed subset of a ring $R$ and $M$ be an $R$-module. For an ideal $I$ of $R$ disjoint with $S$, the following statements are equivalent.

(1) $I(+) M$ is a weakly $S(+) M$-primary ideal of $R(+) M$.

(2) $I(+) M$ is a weakly $S(+) 0$-primary ideal of $R(+) M$.

(3) $I$ is a weakly $S$-primary ideal of $R$ with weakly $S$-primary element $s \in S$ and if $a, b \in R$ with $a b=0$ but $s a \notin \sqrt{I}$ and $s b \notin I$, then $a, b \in \operatorname{ann}_{R}(M)$.

Proof. $(1) \Rightarrow(3)$. Suppose that $(s, m)$ is an $S(+) M$-primary element of $I(+) M$ and $0 \neq a b \in I$ for some $a, b \in R$. Then $(0,0) \neq(a, 0)(b, 0) \in I(+) M$ which implies either $(s, m)(a, 0) \in \sqrt{I(+) M}=\sqrt{I}(+) M$ or $(s, m)(b, 0) \in I(+) M$. Thus, $s a \in \sqrt{I}$ or $s b \in I$ and $I$ is a weakly $S$-primary ideal of $R$. Let $s$ be a weakly $S$-primary element of $I$ and Suppose $a, b \in R$ with $a b=0$ but $s a \notin \sqrt{I}$ and $s b \notin I$. Suppose on contrary that $a \notin a n n_{R}(M)$. Then $a m^{\prime} \neq 0$ for some $m^{\prime} \in M$ and we can write $(0,0) \neq(a, 0)\left(b, m^{\prime}\right) \in I(+) M$. This yields either $(s, m)(a, 0) \in \sqrt{I(+) M}=$ $\sqrt{I}(+) M$ or $(s, m)\left(b, m^{\prime}\right) \in I(+) M$ and so we get $s a \in \sqrt{I}$ or $s b \in I$, a contradiction. Thus $a \in \operatorname{ann}_{R}(M)$. Similarly, we conclude $b \in a n n_{R}(M)$.

$(3) \Rightarrow(2)$. Suppose that $(0,0) \neq\left(a, m_{1}\right)\left(b, m_{2}\right) \in I(+) M$ for some $\left(a, m_{1}\right),\left(b, m_{2}\right) \in$ $R(+) M$. If $a b \neq 0$, then $s a \in \sqrt{I}$ or $s b \in I$, and it is easy to see that $(s, 0)$ is an $S(+) 0$-primary element of $I(+) M$. Next, assume that $a b=0$ but neither $s a \in \sqrt{I}$ nor $s b \in I$. From our assumption (3), $a, b \in \operatorname{ann}_{R}(M)$ and hence $\left(a, m_{1}\right)\left(b, m_{2}\right)=\left(a b, a m_{2}+b m_{1}\right)=(0,0)$, a contradiction.

We note that if the conditions on $I$ and $M$ in (3) of Proposition 12 does not hold, then we may find a weakly $S$-primary ideal of a $\operatorname{ring} R$ such that $I(+) M$ is not weakly $S(+) 0$-primary in $R(+) M$. For example, while $\langle\overline{0}\rangle$ is weakly $S_{5}$-primary in $\mathbb{Z}_{30}$, the ideal $\langle\overline{0}\rangle(+) \mathbb{Z}_{30}$ is not weakly $S_{5}(+) 0$-primary in $\mathbb{Z}_{30}(+) \mathbb{Z}_{30}$. Indeed, $(0,0) \neq(3,1)(10,1) \in\langle\overline{0}\rangle(+) \mathbb{Z}_{30}$ but $(s, 0)(3,1) \notin \sqrt{\langle\overline{0}\rangle(+) \mathbb{Z}_{30}}=\langle\overline{0}\rangle(+) \mathbb{Z}_{30}$ and $(s, 0)(10,1) \notin\langle\overline{0}\rangle(+) \mathbb{Z}_{30}$ for all $s \in S_{5}$.

For rings $R$ and $R^{\prime}$, let $f: R \rightarrow R^{\prime}$ be a ring homomorphism and $J$ be an ideal of $R^{\prime}$. The amalgamation of $R$ and $R^{\prime}$ along $J$ with respect to $f$ is the subring $R \ltimes^{f} J=\{(a, f(a)+j): a \in R, j \in J\}$ of $R \times R^{\prime}$. The amalgamated duplication of a ring $R$ along an ideal $J$ is $R \ltimes J=R \ltimes^{I d_{R}} J=\{(r, r+j): r \in R, j \in J\}$ corresponds to the identity homomorphism $I d_{R}: R \rightarrow R$. In [8], [9], we can see many properties of this ring.

For an ideal $I$ of $R$ and an ideal $K$ of $f(R)+J$, two corresponding ideals of $R \ltimes^{f} J$ can be defined, [9]: $I \ltimes^{f} J=\{(i, f(i)+j): i \in I, j \in J\}$ and $\bar{K}^{f}=\{(a, f(a)+j)$ : $a \in R, j \in J, f(a)+j \in K\}$. For a multiplicatively closed subset $S$ of $R$, one can easily verify that $S \ltimes^{f} J=\{(s, f(s)+j): s \in S, j \in J\}$ and $W=\{(s, f(s)): s \in S\}$ are multiplicatively closed subsets of $R \ltimes^{f} J$. 
The proof of the following lemma can be follows by simple computations.

Lemma 1. Let $R, R^{\prime}, I, K$ and $f$ be as above. Then $\sqrt{I \ltimes^{f} J}=\sqrt{I} \ltimes^{f} J$ and $\sqrt{\bar{K}^{f}}=\overline{\sqrt{K}}^{f}$.

Next, we determine when the ideal $I \ltimes^{f} J$ is $\left(S \ltimes^{f} J\right)$-primary ideal in $R \ltimes^{f} J$.

Theorem 6. Consider the amalgamation of rings $R$ and $R^{\prime}$ along the ideal $J$ of $R^{\prime}$ with respect to a homomorphism $f$. Let $S$ be a multiplicatively closed subset of $R$ and $I$ be an ideal of $R$ disjoint with $S$. Then the following are equivalent.

(1) $I \ltimes^{f} J$ is a weakly $W$-primary ideal of $R \ltimes^{f} J$.

(2) $I \ltimes^{f} J$ is a weakly $\left(S \ltimes^{f} J\right)$-primary ideal of $R \ltimes^{f} J$.

(3) $I$ is a weakly $S$-primary ideal of $R$ and for $a, b \in R$ and a weakly $S$-primary element $s \in S$ with $a b=0$ but $s a \notin \sqrt{I}, s b \notin I$, then $f(a) j+f(b) i+i j=0$ for every $i, j \in J$.

Proof. First we note that $\left(S \ltimes^{f} J\right) \cap\left(I \ltimes^{f} J\right)=\emptyset$ if and only if $W \cap\left(I \ltimes^{f} J\right)=\emptyset$ if and only if $S \cap I=\emptyset$.

$(1) \Rightarrow(2)$. Clear from Remark 1 since $W \subseteq\left(S \ltimes^{f} J\right)$.

$(2) \Rightarrow(3)$. Suppose $I \ltimes^{f} J$ is a weakly $\left(S \ltimes^{f} J\right)$-primary ideal of $R \ltimes^{f} J$ and choose a weakly $\left(S \ltimes^{f} J\right)$-primary element $(s, f(s)+j)$ of $I \ltimes^{f} J$. Let $a, b \in R$ such that $0 \neq a b \in I$ and $s a \notin \sqrt{I}$. Then $(0,0) \neq(a, f(a))(b, f(b)) \in I \ltimes^{f} J$ and by Lemma 1 , we have $(s, f(s)+j)(a, f(a)) \notin \sqrt{I \ltimes^{f} J}$. Hence, $(s, f(s)+j)(b, f(b)) \in I \ltimes^{f} J$ and so $s b \in I$. Therefore, $I$ is a weakly $S$-primary ideal of $R$. Now, let $s$ be a weakly $S$-primary element of $I$ and $a, b \in R$ such that $a b=0, s a \notin \sqrt{I}$ and $s b \notin I$. Then for every $i, j \in J$, we have $(a, f(a)+i)(b, f(b)+j) \in I \ltimes^{f} J$, $(s, f(s)+i)(a, f(a)+i) \notin \sqrt{I \ltimes^{f} J}$ and $(s, f(s))(b, f(b)) \notin I \ltimes^{f} J$. Therefore, we get $f(a) j+f(b) i+i j=(f(a)+i)(f(b)+j)=0$ since $I \ltimes^{f} J$ is weakly $\left(S \ltimes^{f} J\right)$ primary.

$(3) \Rightarrow(1)$. Let $\left(a, f(a)+j_{1}\right),\left(b, f(b)+j_{1}\right) \in R \ltimes^{f} J$ such that $(0,0) \neq(a, f(a)+$ $\left.j_{1}\right)\left(b, f(b)+j_{2}\right)=\left(a b,\left(f(a)+j_{1}\right)\left(f(b)+j_{2}\right)\right) \in I \ltimes^{f} J$. If $a b \neq 0$, then $s a \in \sqrt{I}$ or $s b \in I$ for some weakly $S$-primary element $s$ of $I$. Hence, $(s, f(s))(a, f(a)+j) \in$ $\sqrt{I} \ltimes^{f} J=\sqrt{I \ltimes^{f} J}$ or $(s, f(s))(b, f(b)+j) \in I \ltimes^{f} J$ as required. Now, suppose $a b=0$. Then $f(a) j+f(b) i+i j \neq 0$ and so by assumption either $s a \in \sqrt{I}$ or $s b \in I$. Hence, again $(s, f(s))(a, f(a)+j) \in \sqrt{I} \ltimes^{f} J=\sqrt{I \ltimes^{f} J}$ or $(s, f(s))(b, f(b)+j) \in$ $I \ltimes^{f} J$. Therefore, $I \ltimes^{f} J$ is a weakly $W$-primary ideal of $R \ltimes^{f} J$.

Corollary 5. Let $R, R^{\prime}, J, f$ and $S$ be as in Theorem 6. Then any weakly $\left(S \ltimes^{f} J\right)$ primary ideals of $R \ltimes^{f} J$ containing $\{0\} \times J$ is of the form $I \ltimes^{f} J$ where $I$ is a weakly $S$-primary ideal of $R$.

Proof. Let $K$ be a weakly $\left(S \ltimes^{f} J\right)$-primary ideal of $R \ltimes^{f} J$ containing $\{0\} \times J$. Consider the surjective homomorphism $\varphi: R \ltimes^{f} J \rightarrow R$ defined by $\varphi(a, f(a)+j)=$ $a$. Then $\operatorname{Ker}(\varphi)=\{0\} \times J \subseteq K$ and so $I:=\varphi(K)$ is a weakly $S$-primary ideal of $R$ by Proposition 10. Since $\{0\} \times J \subseteq K$, we conclude that $K=I \ltimes^{f} J$. Moreover, $I \ltimes^{f} J$ is a weakly $\left(S \ltimes^{f} J\right)$-primary ideal of $R \ltimes^{f} J$ for any weakly $S$-primary ideal $I$ of $R$ by Theorem 6 .

Let $T$ be a multiplicatively closed subset of $R^{\prime}$. Then clearly, the set $\bar{T}^{f}=$ $\{(s, f(s)+j): s \in R, j \in J, f(s)+j \in T\}$ is a multiplicatively closed subset of $R \ltimes^{f} J$. 
Theorem 7. Consider the amalgamation of rings $R$ and $R^{\prime}$ along the ideals $J$ of $R^{\prime}$ with respect to an epimorphism $f$. Let $K$ be an ideal of $R^{\prime}$ and $T$ be a multiplicatively closed subset of $R^{\prime}$ disjoint with $K$. The following are equivalent.

(1) $\bar{K}^{f}$ is a weakly $\bar{T}^{f}$-primary ideal of $R \ltimes^{f} J$.

(2) $K$ is a weakly $T$-primary ideal of $R^{\prime}$ and for $f(a), f(b) \in R^{\prime}$ and a weakly $T$-primary element $f(s)$ of $K$, if $(f(a)+j)(f(b)+i)=0^{\prime}$ for every $i, j \in J$, $f(s)(f(a)+j) \notin \sqrt{K}$ and $f(s)(f(b)+i) \notin K$, then $a b=0$.

Proof. One can easily verify that $T \cap K=\phi$ if and only if $\bar{T}^{f} \cap \bar{K}^{f}=\phi$.

$(1) \Rightarrow(2)$. Suppose $\bar{K}^{f}$ is a weakly $\bar{T}^{f}$-primary ideal of $R \ltimes^{f} J$ and choose a weakly $\bar{T}^{f}$-primary element $(s, f(s)+j)$ of $\bar{K}^{f}$. Let $a^{\prime}, b^{\prime} \in R^{\prime}$ such that $0^{\prime} \neq a^{\prime} b^{\prime} \in K$, say, $a^{\prime}=f(a)$ and $b^{\prime}=f(b)$ for $a, b \in R$. Then $(a, f(a)),(b, f(b)) \in R \ltimes^{f} J$ with $(0,0) \neq(a, f(a))(b, f(b))=(a b, f(a b)) \in \bar{K}^{f}$. By assumption, we have either $(s, f(s)+j)(a, f(a))=(s a,(f(s)+j) f(a)) \in \sqrt{\bar{K}}^{f}=\overline{\sqrt{K}}^{f}$ or $(s, f(s)+j)(b, f(b))=$ $(s b,(f(s)+j) f(b)) \in \bar{K}^{f}$. Thus, $f(s)+j \in T$ and $(f(s)+j) f(a) \in \sqrt{K}$ or $(f(s)+j) f(b) \in K$. It follows that $K$ is a weakly $T$-primary ideal of $R^{\prime}$. Now, let $f(s)$ be a weakly $T$-primary element of $K$ and $f(a), f(b) \in R^{\prime}$ such that $(f(a)+$ $j)(f(b)+i)=0^{\prime}, f(s)(f(a)+j) \notin \sqrt{K}$ and $f(s)(f(b)+i) \notin K$. Since $\bar{K}^{f}$ is weakly $\bar{T}^{f}$-primary and for every $i, j \in J,(a, f(a)+j)(b, f(b)+i) \in \bar{K}^{f}$, then we must have $a b=0$ and we are done.

$(2) \Rightarrow(1)$. Choose a weakly $T$-primary element $f(s)$ of $K$. Let $(0,0) \neq(a, f(a)+$ $\left.j_{1}\right)\left(b, f(b)+j_{2}\right)=\left(a b,\left(f(a)+j_{1}\right)\left(f(b)+j_{2}\right)\right) \in \bar{K}^{f}$ for $\left(a, f(a)+j_{1}\right),(b, f(b)+$ $\left.j_{2}\right) \in R \ltimes^{f} J$. Then $\left(f(a)+j_{1}\right)\left(f(b)+j_{2}\right) \in K$. If $\left(f(a)+j_{1}\right)\left(f(b)+j_{2}\right) \neq 0^{\prime}$, then $f(s)\left(f(a)+j_{1}\right) \in \sqrt{K}$ or $f(s)\left(f(b)+j_{2}\right) \in K$. Thus, $(s, f(s))(a, f(a)+$ $\left.\left.j_{1}\right)\right)=\left(s a, f(s a)+j_{1} f(s)\right) \in \overline{\sqrt{K}}^{f}=\sqrt{\bar{K}^{f}}$ or $\left.(s, f(s))\left(b, f(b)+j_{2}\right)\right) \in \bar{K}^{f}$ and the result follows. Suppose $\left(f(a)+j_{1}\right)\left(f(b)+j_{2}\right)=0^{\prime}$. Then $a b \neq 0$ and so by our assumption, we conclude $f(s)\left(f(a)+j_{1}\right) \in \sqrt{K}$ or $f(s)\left(f(b)+j_{2}\right) \in K$. Hence, again $\left.(s, f(s))\left(a, f(a)+j_{1}\right)\right) \in \sqrt{\bar{K}^{f}}$ or $\left.(s, f(s))\left(b, f(b)+j_{2}\right)\right) \in \bar{K}^{f}$ and so $\bar{K}^{f}$ is a weakly $\bar{T}^{f}$-primary ideal of $R \ltimes^{f} J$.

In particular, if $S$ is a multiplicatively closed subset $S$ of $R, S \times f(S)$ is also a multiplicatively closed subset of $R \ltimes^{f} J$. Hence, we have the following corollary of Theorem 7 .

Corollary 6. Let $R, R^{\prime}, J, S$ and $f$ be as in Theorem 6 . Let $K$ be an ideal of $R^{\prime}$ and $T=f(S)$. Then the following statements are equivalent.

(1) $\bar{K}^{f}$ is a weakly $(S \times T)$-primary ideal of $R \ltimes^{f} J$.

(2) $\bar{K}^{f}$ is a weakly $\bar{T}^{f}$-primary ideal of $R \ltimes^{f} J$.

(3) $K$ is a weakly $T$-primary ideal of $R$ and for $f(a), f(b) \in R^{\prime}$ and a weakly $T$-primary element $f(s)$ of $K$, if $(f(a)+j)(f(b)+i)=0^{\prime}$ for every $i, j \in J$, $f(s)(f(a)+j) \notin \sqrt{K}$ and $f(s)(f(b)+i) \notin K$, then $a b=0$.

\section{S-WEAKLY LASKERIAN RINGS}

It is well-know that a primary decomposition of an ideal $I$ of a $\operatorname{ring} R$ is an expression of $I$ as a finite intersection of primary ideals of $R$. In this case, we say that $I$ is decomposable in $R$. Recall that a $\operatorname{ring} R$ is called Laskerian if every proper ideal of $R$ is decomposable. More general, in [19], $S$-decomposable ideals are defined as those ideals that can be written as a finite intersection of $S$-primary ideals. A 
ring $R$ is called $S$-Laskerian if for every proper ideal $I$ of $R$, either $I \cap S \neq \phi$ or $(I: s)$ is $S$-decomposable for some $s \in S$. Analogous to these definitions, we have the following.

Definition 2. Let $S$ be a multiplicatively closed subset of a ring $R$ and $I$ be a proper ideal of $R$.

(1) $I$ is called weakly decomposable if it can be expressed as a finite intersection of weakly primary ideals. Moreover, $R$ is called weakly Laskerian if every proper ideal of $R$ is weakly decomposable.

(2) $I$ is called weakly $S$-decomposable if it can be expressed as a finite intersection of weakly $S$-primary ideals. Moreover, $R$ is called $S$-weakly Laskerian if for every proper ideal $I$ of $R$, either $I \cap S \neq \phi$ or $(I: s)$ is weakly $S$-decomposable for some $s \in S$.

While clearly every decomposable ( $S$-decomposable) ideal is weakly decomposable (weakly $S$-decomposable), the converse need not be true. For example, the zero ideal of the ring of real valued continuous functions $C[0,1]$ is clearly weakly decomposable which is not decomposable, [5, Exercise 6, page 55].

In this section, we only give some basic results concerning these concepts. Motivated and inspired by S-decomposable ideals introduced in [19], many other definitions and results will be introduced and studied in a future coming research.

Proposition 13. Let $S \subseteq \operatorname{reg}(R)$ be a multiplicatively closed subset of a ring $R$. If $R$ is weakly Laskerian, then it is $S$-weakly Laskerian.

Proof. Suppose $R$ is weakly Laskerian and let $I$ be an ideal of $R$ such that $I \cap S=\phi$. Then $I=\bigcap_{i=1}^{n} Q_{i}$ where $Q_{i}$ is weakly primary for all $i$. Since $\left(\bigcap_{i=1}^{n} Q_{i}\right) \cap S=\phi$, then $Q_{j} \cap S=\phi$ for at least one $j \in\{1,2, \cdots, n\}$. Now, suppose with no loss of generality that $Q_{i} \cap S=\phi$ for $i=1,2, \cdots, k$ and $Q_{i} \cap S \neq \phi$ for $i=k+1, k+2, \cdots, n$. Then for all $i=k+1, k+2, \cdots, n$, clearly there exists $s \in S$ such that $s \in Q_{i}$ and so $\left(Q_{i}: s\right)=R$ Moreover, as $S \subseteq \operatorname{reg}(R),\left(Q_{i}: s\right)$ is weakly primary (and so weakly $S$-primary) for $i=1,2, \cdots, k$. Therefore, $(I: s)=\left(\bigcap_{i=1}^{n} Q_{i}: s\right)=\bigcap_{i=1}^{n}\left(Q_{i}: s\right)=$ $\bigcap_{i=1}^{k}\left(Q_{i}: s\right)$ is a weakly $S$-primary decomposition and $R$ is $S$-weakly Laskerian.

Proposition 14. Let $S \subseteq \operatorname{reg}(R)$ be a multiplicatively closed subset of a ring $R$ and $I$ be an ideal of $R$ disjoint with $S$.

(1) If $(I: s)$ is weakly $S$-decomposable for some $s \in S$, then $S^{-1} I$ is weakly decomposable in $S^{-1} R$ and $S^{-1} I \cap R=\left(I: s^{\prime}\right)$ for some $s^{\prime} \in S$.

(2) The converse of (1) is true if each term in the decomposition of $S^{-1} I$ is not primary.

Proof. (1) Suppose $(I: s)$ is weakly $S$-decomposable for some $s \in S$, say, ( $I$ : $s)=\bigcap_{i=1}^{n} Q_{i}$ where $Q_{i}$ is weakly $S$-primary for all $i \in\{1,2, \cdots, n\}$. Then $S^{-1} Q_{i}$ is weakly primary and $S^{-1} Q_{i} \cap R=\left(Q_{i}: s_{i}\right)$ for some $s_{i} \in S$ by Corollary 2. Now, by [14, Exercise 4, page 75], we have $S^{-1}(I: s)=\left(S^{-1} I: S^{-1}\langle s\rangle\right)=\left(S^{-1} I\right.$ : $\left.S^{-1} R\right)=S^{-1} I$. Therefore, $S^{-1} I=S^{-1}\left(\bigcap_{i=1}^{n} Q_{i}\right)=\bigcap_{i=1}^{n}\left(S^{-1} Q_{i}\right)$ is a weakly primary 
decomposition of $S^{-1} I$. For the proof of the other part, we let $t=s_{1} s_{2} \cdots s_{n}$ and start by justifying that $\left(Q_{i}: s_{i}\right)=\left(Q_{i}: t\right)$ for all $i \in\{1,2, \cdots, n\}$. Indeed, let $i \in\{1,2, \cdots, n\}$ and $a \in\left(Q_{i}: t\right)$ so that $a s_{1} s_{2} \cdots s_{i-1} s_{i+1} \cdots s_{n} \in\left(Q_{i}: s_{i}\right)$. If $Q_{i}=\langle 0\rangle$, then $(I: s)=\langle 0\rangle$ and so $S^{-1} I \cap R=S^{-1}(I: s) \cap R=S^{-1}\langle 0\rangle \cap$ $R=0_{S}=\left((I: s): s^{\prime}\right)=\left(I: s s^{\prime}\right)$ for some $s \prime \in S$. If $Q_{i}$ is non-zero and as $S \subseteq \operatorname{reg}(R)$, then $\left(Q_{i}: s_{i}\right)$ is weakly primary by (2) of Proposition 4. Since clearly $s_{1} s_{2} \cdots s_{i-1} s_{i+1} \cdots s_{n} \notin \sqrt{\left(Q_{i}: s_{i}\right)}$, then $a \in\left(Q_{i}: s_{i}\right)$ and so $\left(Q_{i}: t\right) \subseteq\left(Q_{i}: s_{i}\right)$. Hence, $\left(Q_{i}: s_{i}\right)=\left(Q_{i}: t\right)$ for all $i \in\{1,2, \cdots, n\}$. Now, $S^{-1} I=\bigcap_{i=1}^{n}\left(S^{-1} Q_{i}\right)$ implies $S^{-1} I \cap R=\bigcap_{i=1}^{n}\left(S^{-1} Q_{i}\right) \cap R=\bigcap_{i=1}^{n}\left(S^{-1} Q_{i} \cap R\right)=\bigcap_{i=1}^{n}\left(Q_{i}: t\right)=\left(\bigcap_{i=1}^{n} Q_{i}: t\right)=$ $((I: s): t)=(I: s t)=\left(I: s^{\prime}\right)$ for some $s^{\prime} \in S$.

(2) Suppose $S^{-1} I=\bigcap_{i=1}^{n}\left(S^{-1} Q_{i}\right)$ where for all $i, S^{-1} Q_{i}$ is weakly primary in $S^{-1} R$ that is not primary. Then $S^{-1} I$ is weakly primary in $S^{-1} R$ by [4, Theorem 2.3]. It follows that $I$ is a weakly $S$-primary ideal of $R$ by Corollary 2. If $I$ is nonzero, then as $S \subseteq \operatorname{reg}(R),\left(I: s^{\prime}\right)$ is weakly primary (and so weakly $S$-primary) in $R$ for some weakly $S$-primary element $s$ of $I$ by (2) of Proposition 4 . Suppose $I=0$. Note that by $\left[4\right.$, Theorem 2.2], $\sqrt{S^{-1} Q_{i}}=\sqrt{S^{-1} 0}$, and so $S^{-1} Q_{i} \cap R$ is weakly primary in $R$ for all $i$. Indeed, if $0 \neq a b \in S^{-1} Q_{i} \cap R$, then $\frac{a}{1} \cdot \frac{b}{1} \in S^{-1} Q_{i}$ and $\frac{a}{1} \cdot \frac{b}{1} \neq \frac{0}{1}$ since otherwise $S \subseteq \operatorname{reg}(R)$ implies $a b=0$, a contradiction. Thus, either $\frac{a}{1} \in$ $\sqrt{S^{-1} Q_{i}}=\sqrt{S^{-1} 0}$ or $\frac{b}{1} \in S^{-1} Q_{i}$. If $\frac{a}{1} \in \sqrt{S^{-1} Q_{i}}$, then $a \in \sqrt{0} \subseteq \sqrt{S^{-1} Q_{i} \cap R}$ as $S \subseteq \operatorname{reg}(R)$. Otherwise, $\frac{b}{1} \in S^{-1} Q_{i}$ implies $b \in S^{-1} Q_{i} \cap R$. Moreover, if there is $s \in S$ such that $s \in\left(S^{-1} Q_{i} \cap R\right) \cap S$, then clearly $Q_{i} \cap S \neq \phi$, a contradiction. Thus, $S^{-1} Q_{i} \cap R$ is a weakly $S$-primary ideal of $R$ for all $i$. By assumption, there is $s^{\prime} \in S$ such that $\left(0: s^{\prime}\right)=S^{-1} 0 \cap R=\bigcap_{i=1}^{n}\left(S^{-1} Q_{i}\right) \cap R=\bigcap_{i=1}^{n}\left(S^{-1} Q_{i} \cap R\right)$. Thus, $\left(0: s^{\prime}\right)$ is weakly $S$-decomposable for some $s^{\prime} \in S$ as needed.

Proposition 15. Let $S \subseteq$ reg $(R)$ be a multiplicatively closed subset of a ring $R$.

(1) If $R$ is $S$-weakly Laskerian, then $S^{-1} R$ is weakly Laskerian and for any ideal $I$ of $R$ with $I \cap S=\phi$, we have $S^{-1} I \cap R=\left(I: s^{\prime}\right)$ for some $s^{\prime} \in S$.

(2) The converse of (1) is true if each term in a weakly primary decomposition of every ideal of $S^{-1} R$ is not primary.

Proof. (1) Suppose $R$ is $S$-weakly Laskerian and let $S^{-1} J$ be a proper ideal of $S^{-1} R$ for some proper ideal $J$ of $R$ with $J \cap S=\phi$. By assumption, $(J: s)$ is weakly $S$-decomposable for some $s \in S$. By (1) of Proposition 14, $S^{-1} J$ is weakly decomposable and so $S^{-1} R$ is weakly Laskerian. Now, let $I$ be any ideal of $R$ with $I \cap S=\phi$. Since $(I: s)$ is weakly $S$-decomposable in $R$ for some $s \in S$, then again by (1) of Proposition 14, we get $S^{-1} I \cap R=\left(I: s^{\prime}\right)$ for some $s^{\prime} \in S$ as required.

(2) Let $I$ be a proper ideal of $R$ and suppose $I \cap S=\phi$. Then $S^{-1} I=\bigcap_{i=1}^{n}\left(S^{-1} Q_{i}\right)$ where $S^{-1} Q_{i}$ is weakly primary in $S^{-1} R$ that is not primary and $S^{-1} I \cap R=\left(I: s^{\prime}\right)$ for some $s^{\prime} \in S$. By the proof of (2) of Proposition 14, we conclude that there exists $s \in S$ such that $(I: s)$ is weakly $S$-decomposable. Therefore, $R$ is $S$-weakly Laskerian. 


\section{REFERENCES}

[1] F. A. Almahdi, E. M. Bouba, M. Tamekkante, On weakly $S$-prime ideals of commutative rings, Analele Stiintifice ale Universitatii Ovidius Constanta, Seria Mathematica (To appear)

[2] D. F. Anderson and A. Badawi, On $n$-absorbing ideals of commutative rings, Comm. Algebra 39 (2011), no. 5, 1646-1672.

[3] D. Anderson and E. Smith, Weakly prime ideals, Houston Journal of Mathematics 29 (4) (2003), 831-840.

[4] S. E. Atani, ,F. Farzalipour. On weakly primary ideals, Georgian Mathematical Journal, 12 (3) (2005), 423-429. https://doi.org/10.1515/GMJ.2005.423.

[5] M.F. Atiyah, I.G. Macdonald, Introduction to Commutative Algebra. Addison-Wesley, Reading (1969).

[6] G. Călugăreanu, UN-rings. Journal of Algebra and Its Applications, 15 (10) (2016), 1650182.

[7] M. D'Anna and M. Fontana, An amalgamated duplication of a ring along an ideal: the basic properties, J. Algebra Appl. 6 (2007), no. 3, 443-459.

[8] M. D'Anna, M. Fontana, The amalgamated duplication of a ring along a multiplicativecanonical ideal, Ark. Mat. 45 (2) (2007), 241-252.

[9] M. D'Anna, C.A. Finocchiaro, and M. Fontana, Properties of chains of prime ideals in an amalgamated algebra along an ideal, J. Pure Appl. Algebra 214 (2010), 1633-1641.

[10] R.W. Gilmer, Multiplicative ideal theory, 12, M. Dekker, 1972.

[11] A. Hamed, S. Hizem, Modules satisfying the $S$-Noethrian property and $S$-accr. Commun. Algebr. 44, (2016) 1941-1951.

[12] A. Hamed, A. Malek, $S$-prime ideals of a commutative ring, Beiträge zur Algebra und Geometrie/Contributions to Algebra and Geometry, 61.(3) (2020), 533-542.

[13] H. A. Khashan, E. Yetkin Celikel, $S$ - $n$-ideals of commutative rings (submitted).

[14] M. D. Larsen, P.J. McCarthy, Multiplicative Theory of Ideals, Academic Press, 1971.

[15] C.Jayaram, U. Tekir, Von Neumann regular modules. Commun. Algebra 46(5) (2018) 22052217. DOI:10.1080/00927872.2017.1372460

[16] J. Von Neumann, On regular rings. Proc. Natl. Acad. Sci. USA. 22 (12) (1936) 707-713. DOI: 10.1073 pnas.22.12.707

[17] E. S. Sevim, I. T Arabacı, U. Tekir and S. Koc, On S-prime submodules, Turkish J. Math. 43 (2019), 1036-1046.

[18] U. Tekir, S. Koc, K.H. Oral, n-ideals of commutative rings, Filomat 31 (10) (2017) 2933-2941.

[19] S. Visweswaran, Some results on $S$-primary ideals of a commutative ring, Beiträge zur Algebra und Geometrie/Contributions to Algebra and Geometry, (2021), 1-20.

[20] F. Wang, H.Kim, Foundations of Commutative Rings and Their Modules. Singapore: Springer, 2016.

Electrical-Electronics Engineering, Faculty of Engineering, HasanKalyoncu University, Gaziantep, Turkey.

E-mail address: ece.celikel@hku.edu.tr, yetkinece@gmail.com.

Department of Mathematics, Faculty of Science, Al al-Bayt University, Al Mafraq, JORDAN.

E-mail address: hakhashan@aabu.edu.jo. 